\title{
Very high order lattice perturbation theory for Wilson loops
}

\section{R. Horsley ${ }^{a}$, G. Hotzel ${ }^{b}$, E.-M. Ilgenfritz ${ }^{c, d}$, Y. Nakamura ${ }^{e}$, H. Perlt ${ }^{* b}$, P. E. L. Rakow ${ }^{f}$, G. Schierholz ${ }^{g}$, A. Schiller ${ }^{b}$}

${ }^{a}$ School of Physics, University of Edinburgh, Edinburgh EH9 3JZ, UK

${ }^{b}$ Institut für Theoretische Physik, Universität Leipzig, 04109 Leipzig, Germany

${ }^{c}$ Fakultät für Physik, Universität Bielefeld, 33501 Bielefeld, Germany

${ }^{d}$ Institut für Physik, Humboldt-Universität zu Berlin, 12489 Berlin, Germany

${ }^{e}$ Institut für Theoretische Physik, Universität Regensburg, 93040 Regensburg, Germany

${ }^{f}$ Theoretical Physics Division, Department of Mathematical Sciences, University of Liverpool,

Liverpool L69 3BX, UK

${ }^{g}$ Deutsches Elektronen-Synchrotron DESY, 22603 Hamburg, Germany

E-mail:perlt@itp.uni-leipzig.de

\begin{abstract}
We calculate perturbative Wilson loops of various sizes up to loop order $n=20$ at different lattice sizes for pure plaquette and tree-level improved Symanzik gauge theories using the technique of Numerical Stochastic Perturbation Theory. This allows us to investigate the behavior of the perturbative series at high orders. We observe differences in the behavior of perturbative coefficients as a function of the loop order. Up to $n=20$ we do not see evidence for the often assumed factorial growth of the coefficients. Based on the observed behavior we sum this series in a model with hypergeometric functions. Alternatively we estimate the series in boosted perturbation theory. Subtracting the estimated perturbative series for the average plaquette from the non-perturbative Monte Carlo result we estimate the gluon condensate.
\end{abstract}

The XXVIII International Symposium on Lattice Field Theory

June 14-19,2010

Villasimius, Sardinia Italy

\footnotetext{
*Speaker.
} 


\section{Introduction}

Since the introduction of the non-perturbative gluon condensate by Shifman, Vainshtein and Zakharov [1] there have been many attempts to obtain reliable numerical results for this quantity. Soon it became clear that lattice gauge theory provides a promising tool to calculate it from Wilson loops $W_{N M}$ of sizes $N \times M$. In [2,3] the plaquette was used whereas larger Wilson loops have been investigated in $[4,5]$. In all cases it turned out to be crucial to know the perturbative tail of the Wilson loops as precisely as possible. In the last decade the application of Numerical Stochastic Perturbation Theory (NSPT) [6] pushed the perturbative order of the plaquette up to order $n=10$ [7] and even $n=16$ [8].

Apart from the extraction of the gluon condensate there is a general interest in the behavior of perturbative series in QCD (for a recent investigation see [9]). It is generally believed that these series are asymptotic, and assumed that for large $n$ the leading growth of the coefficients $a_{n}$ is factorial. Using the technique of NSPT one reaches orders of the perturbative series where a possible set-in of this assumed behavior can be tested - at least for finite lattices. In a more recent paper [10] Narison and Zakharov discussed the difference between short and long perturbative series and its impact on the determination of the gluon condensate.

In order to test to what extent $\mathscr{O}\left(a^{2}\right)$ improvement influences the behavior of the series we used in addition to the standard Wilson plaquette gauge action the tree-level improved Symanzik gauge action obtained by Weisz implementing Symanzik's improvement programme [11].

We present perturbative calculations in NSPT up to order $n=20$ for Wilson loops using the Wilson action for lattice sizes $L^{4}$ with $L=4,6,8,12$. In case of the Symanzik action we have computed the $W_{N M}$ for $L=4,6,8,10$. The computation for $L=12$ were performed on a NEC SX-9 computer of RCNP at Osaka University, for $L=10$ at the HLRN Berlin/Hannover, all others on Linux/HP - clusters at Leipzig University.

\section{NSPT calculation up to $n=20$}

NSPT allows perturbative calculations on a finite lattice up to loop order $n$ which practically cannot be reached by the standard diagrammatic approach. A limit is set only by storage limitations and machine precision. The algorithm is introduced and discussed in detail in $[6,12]-$ we will not present it in this paper. For a detailed discussion of our results we refer to a forthcoming paper [13].

In order to fix the notation we write the general expansion of a Wilson loop of size $N \times M$ in terms of the bare lattice coupling $g$ as

$$
W_{N M}=\sum_{n=0}^{20} W_{N M}^{(n)} g^{2 n}
$$

The coefficients $W_{N M}^{(n)}$ are determined with NSPT and need to be known to a very good precision. In Figure 1 we show some results for these coefficients using the Wilson and Symanzik actions. One can see from that Figure that the statistical errors within the NSPT algorithm are rather small.

At all $n$ all the loops behave rather similarly, with the coefficents decreasing smoothly, in a similar way for all loop sizes. At smaller $n$ we see that the coefficients change sign and magnitude in a rather unpredictable way, particularly for large loops. We recognize some remarkable 



Figure 1: Selected $W_{N M}^{(n)}$ for $L=12$ and Wilson action (left) and for $L=10$ and Symanzik action (right). Positive/negative signs of the coefficients are given by open/full symbols.

differences in the behavior between the two actions. First, for the Symanzik action the sign change in the expansion coefficients sets in at smaller Wilson loop sizes $N \times M$ and is extended to larger loop orders $n$. Second, the values of the Symanzik coefficients themselves are much smaller than their Wilson action counterparts. However, concerning the relative convergence behavior of the perturbative series (2.1) one should have in mind that the coupling $g^{2}$ used in computing physical observables is larger in the Symanzik case than in the Wilson case. At larger $n$ a seemingly asymptotic behavior (without sign changes) emerges.

One essential test for the validity of our perturbative calculations with NSPT is the signal/noise ratio. The expansion of the (integer) loop-order $n$ is constructed from even powers $g^{2 n}$. Likewise one can build non-loop contributions which are half-integer and combined from odd powers of $g$ they should vanish. In Fig. 2 we show that the loop contributions are always clearly separated in magnitude from the non-loop (noise) terms.

In addition we have to raise the question about the infinite volume limit $L \rightarrow \infty$ of the loop series. This is related to the definition of the gluon condensate which is clearly a $L=\infty$ quantity. We follow the approach to extrapolate each coefficient at finite $L$ from $W_{N M, L}^{(n)}$ to $W_{N M, \infty}^{(n)}$ using the ansatz

$$
W_{N M, L}^{(n)}=a_{N M, n}+b_{N M, n} L^{-4}+c_{N M, n} \log (L) L^{-6} .
$$

\section{Perturbative series at large order}

It is generally assumed that perturbative series in continuum QCD are asymptotic. The situation might be different for perturbative series on finite lattices. Here we have both ultraviolet and infrared cut-offs and the series could be summed up to a finite value. With our computed coefficients up to order $n=20$ we are able to check this conjecture to a so far unrivaled level.

To perform such a summation we propose a model which is a generalization of the ansatz used in [14]. Denoting the generic coefficients of the perturbative series as $c_{n}$ we have found that a large set of our data above some loop order $n_{0}$ can be described rather well by the following ansatz for 

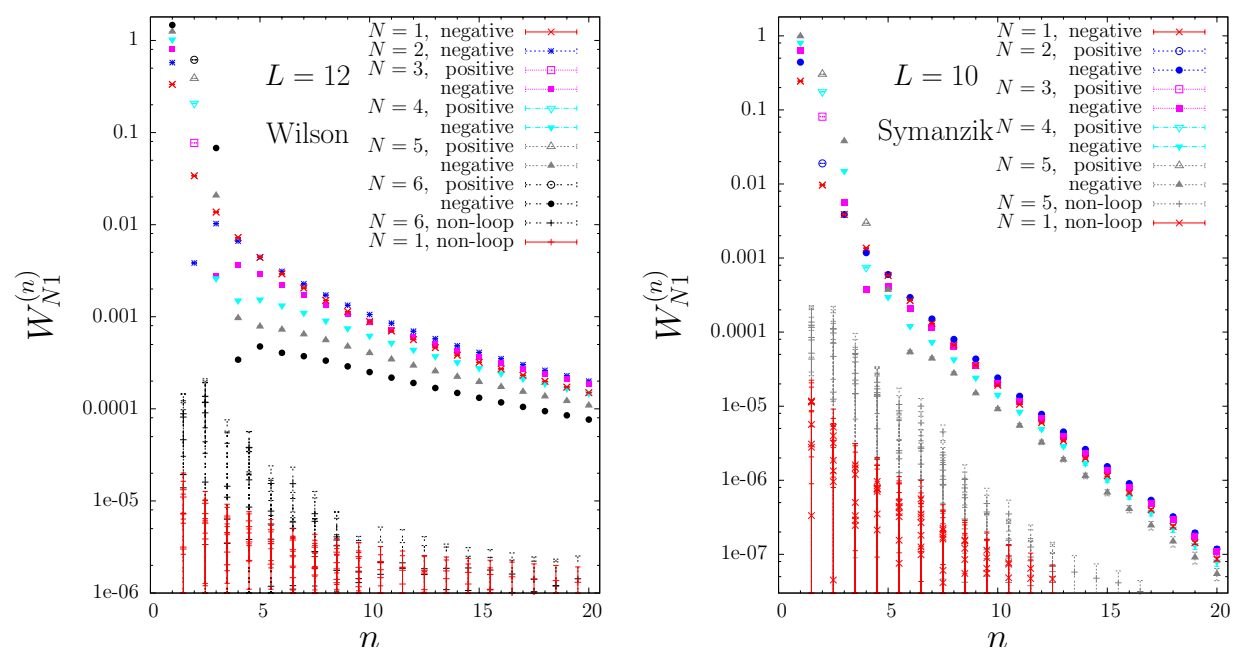

Figure 2: Selected $W_{N 1}^{(n)}$ versus $n$ together with typical sizes (in magnitude) of non-loop contributions. Positive/negative signs of the coefficients are given by open/full symbols. Left: $L=12$ and Wilson gauge action, right: $L=10$ and Symanzik action. Points at half-integer $n$ (the non-loop terms) are pure noise - they show a good signal/noise ratio.

the ratio of subsequent coefficients

$$
r_{n}=c_{n} / c_{n-1}=u\left(1-\frac{1+\gamma}{n}\right)+\frac{p}{n(n+s)}, \quad n>n_{0},
$$

where $u, \gamma, p, s$ are free parameters to be fitted. The series based on (3.1) can be summed up to $n=\infty$ for $g^{2}<1 / u$. For $n_{0}=1$ we obtain as solution

$$
\begin{aligned}
W_{N M, \infty} & =\frac{(s+1) W_{N M}^{(1)}{ }_{2} F_{1}\left((1+s-\gamma) / 2-\tau,(1+s-\gamma) / 2+\tau ; s+1 ; g^{2} u\right)}{p-(s+1) u \gamma}, \\
\tau & =\frac{1}{2} \sqrt{\left((\gamma+s+1)^{2} u-4 p\right) / u}
\end{aligned}
$$

As final hypergeometric model at $n_{0}=1$ we use

$$
W_{N M, H Y P}=W_{N M, \infty}-\left(c_{1, H Y P}-W_{N M}^{(1)}\right) g^{2},
$$

where $c_{1, H Y P}$ is the coefficient of $g^{2}$ in the corresponding $g^{2}$-expansion of $W_{N M, \infty}$. For other values of $n_{0}$ the formulae (3.2) and (3.3) change accordingly. In Fig. 3 we show two example plots for the ratio $r_{n}$ together with the model fit based on (3.1). In all investigated cases we do not observe a factorial growth of the coefficients up to order $n=20$. Formally, one can use model (3.3) (used for finite lattices) also for the $L \rightarrow \infty$ extrapolated coefficients.

A possible alternative method consists in applying boosted perturbation theory, i.e. a rearrangement of the series in terms of a boosted coupling $g_{b}[8]\left(W_{11} \equiv P\right)$. Using the summed perturbative series $P_{n^{\star}}\left(g^{2}\right)=1+\sum_{n=1}^{n^{\star}} W_{11}^{(n)} g^{2 n}$ we get the boosted summed plaquette $P_{20, b}\left(g_{b}\right)$ as follows

$$
g^{2} \rightarrow g_{b}^{2}=\frac{g^{2}}{P_{20}(g)} \quad \rightarrow \quad P_{20, b}\left(g_{b}\right)=1+\sum_{n=1}^{20} W_{b, 11}^{(n)} g_{b}^{2 n}
$$



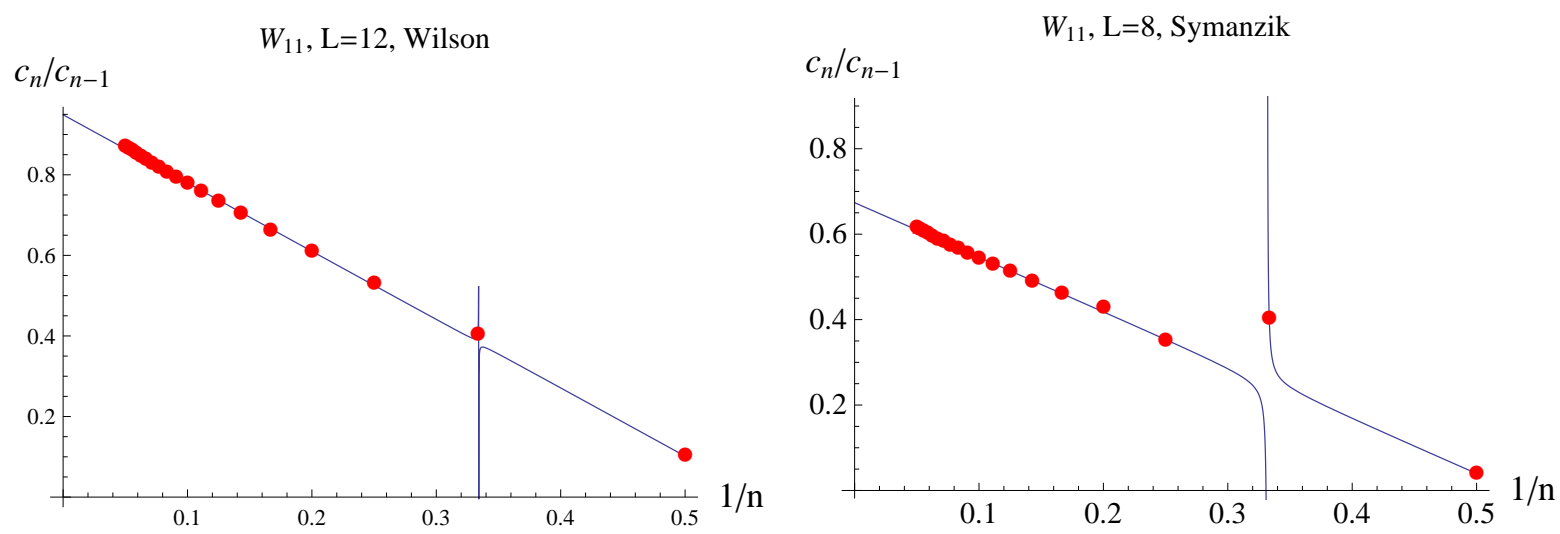

Figure 3: Domb-Sykes plots for $W_{11}$. Left: $L=12$ and Wilson action, right: $L=8$ and Symanzik action.

In Fig. 4 we compare the summed perturbative series $P_{n^{\star}}(g)$ with $P_{n^{\star}, b}\left(g_{b}\right)$ as function of maximal loop order $n^{\star}$ for $L=12$. One clearly recognizes a distinct plateau reached by boosting for $n^{\star}<20$. The naive series is far from the model value computed from (3.3). Although this behavior of the boosted perturbation theory is found for finite $L$, we assume that it remains valid also in the infinite volume limit.
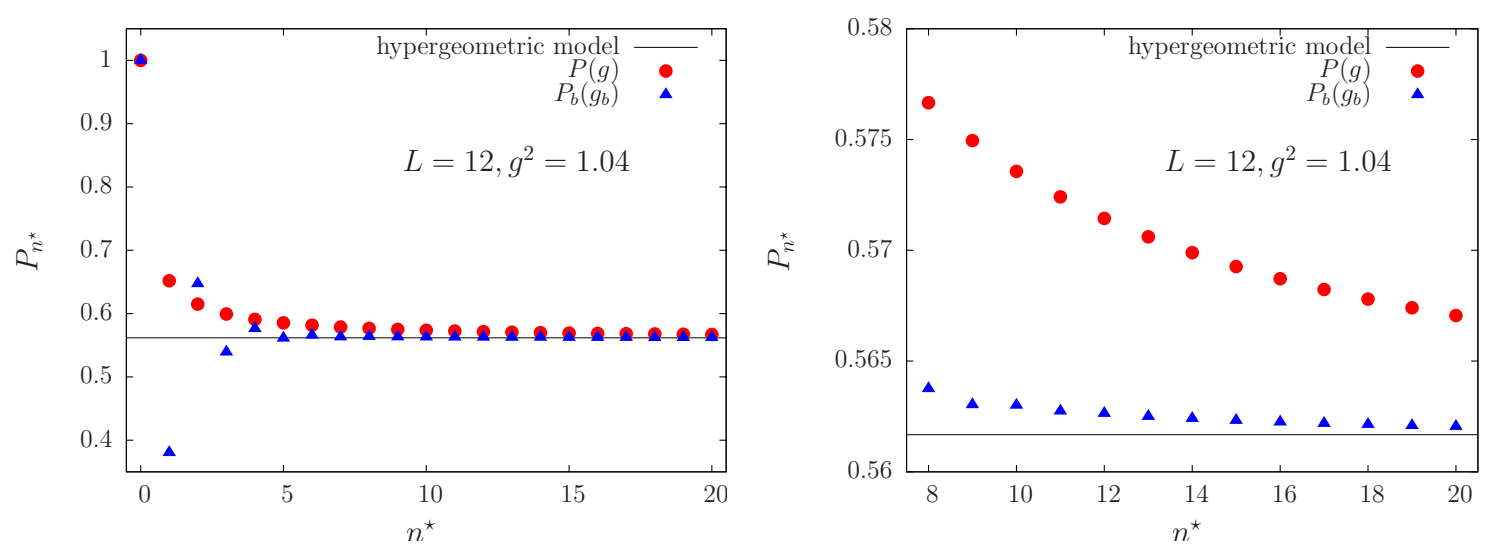

Figure 4: Perturbative plaquette $P_{n^{\star}}$ as function of loop order $n^{\star}$ for naive and boosted series; left: all orders, right: zoomed in by the choice $8 \leq n^{\star} \leq 20$. The choice $g^{2}=1.04$ corresponds to the largest coupling where the solution (3.2) remains real.

\section{Non-perturbative gluon condensate}

Having under control the large order perturbative series we estimate now the non-perturbative gluon condensate $\left\langle\left(\alpha_{s} / \pi\right) G G\right\rangle$. Being a physical quantity we should use the $L=\infty$ extrapolated values of the perturbative coefficients. On the other hand it could be useful to get numbers for the "gluon condensate" at finite $L$ also: they provide some numerical insight how the infinite volume limit is approached.

We use the relation between the plaquette measured in Monte Carlo simulations $P_{M C}$ and its 
perturbative analogue $P$ :

$$
P_{M C}=P+a^{4} \frac{\pi^{2}}{36}\left(\frac{b_{0} g^{2}}{\beta(g)}\right)\left\langle\frac{\alpha_{s}}{\pi} G G\right\rangle,
$$

which defines the gluon condensate. In (4.1) $\beta(g)$ denotes the standard $\beta$-function with $b_{0}$ being its leading coefficient. From (4.1) it is obvious that the precision of the difference $\Delta P=P-P_{M C}$ determines the reliability of an estimate for $\left\langle\frac{\alpha_{s}}{\pi} G G\right\rangle$.

It has been proposed [10] to extract the gluon condensate taking into account a perturbative series of the plaquette summed to an order $n^{\star}\left(P_{n^{\star}}\right)$ from the following ansatz

$$
a^{4} \frac{\pi^{2}}{36}\left(\frac{-b_{0} g^{2}}{\beta(g)}\right)\left\langle\frac{\alpha_{s}}{\pi} G G\right\rangle=\Delta P_{n^{\star}}=P_{n^{\star}}-P_{M C},
$$

where $\Delta P_{n^{\star}}$ contains possible $a^{2}$ and $a^{4}$ contributions

$$
\Delta P_{n^{\star}}=c_{2}\left(n^{\star}\right) a^{2}+c_{4}\left(n^{\star}\right) a^{4} .
$$

The authors in [10] argued that non-zero values of the coefficient $c_{2}\left(n^{\star}\right)$ are an artefact due to a truncation of the series: above some value of $n^{\star}$ this coefficient should vanish. Our perturbative series for both, the naive series (finite $L$ ) and boosted series $(L=\infty)$ confirm their hypothesis: for boosted perturbation theory the $a^{2}$-term decreases rapidly to zero for $n^{\star}>10$ (see left of Fig. 5); for naive perturbation theory and finite $L$ one has to sum the series to much larger $n^{\star}$.
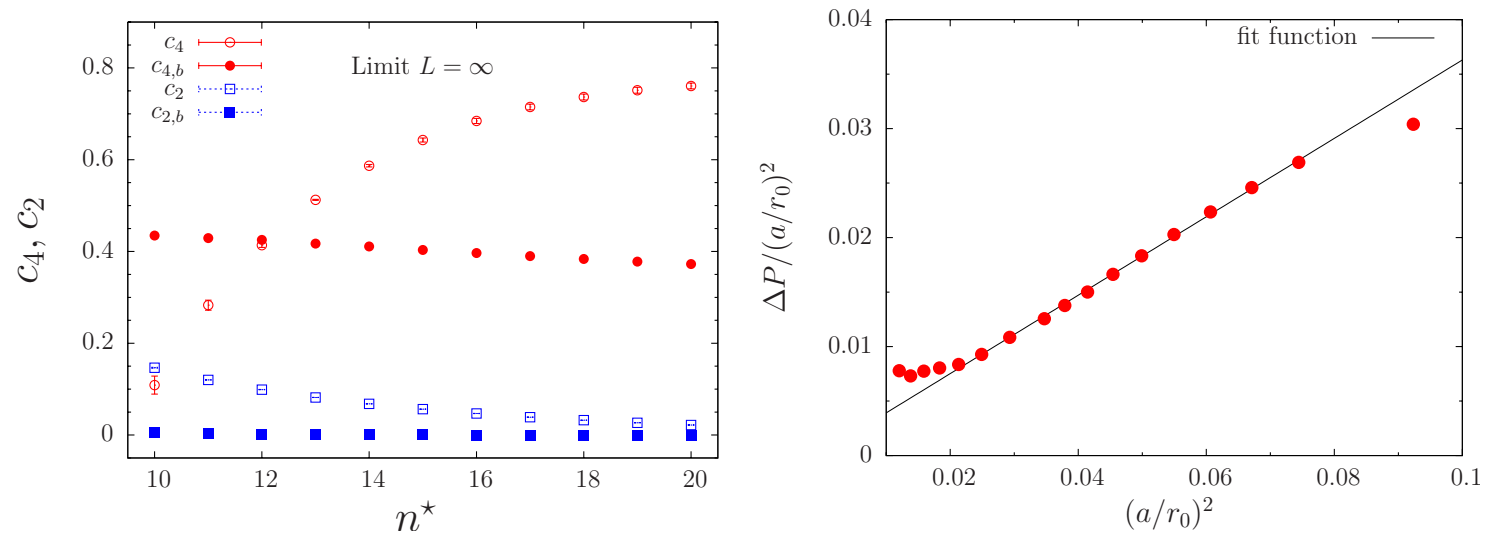

Figure 5: Left: Comparison of $c_{4}\left(n^{\star}\right)$ and $c_{2}\left(n^{\star}\right)$ for naive and boosted $(b)$ perturbation theory in the extrapolated limit $L=\infty$. Right: $\Delta P /\left(a / r_{0}\right)^{2}$ as function of $\left(a / r_{0}\right)^{2}$ for boosted perturbation theory. The line is a linear fit in $a^{2}$ for the range $0.02 \leq\left(a / r_{0}\right)^{2} \leq 0.08, r_{0}$ denotes the Sommer scale.

From the results obtained so far we conclude that the most reliable estimate for the gluon condensate can be obtained for the Wilson action using boosted perturbation theory. In the right of Figure 5 we show $\Delta P /\left(a / r_{0}\right)^{2}$ as function of $\left(a / r_{0}\right)^{2}$ using boosted perturbation theory with $n^{\star}=20$ and our own non-perturbative plaquette measurements at different $\beta$ values and lattice sizes for $r_{0} / a \geq 2.5$. We clearly recognize a dominant linear $a^{2}$ behavior. The necessary conversion $\beta \leftrightarrow\left(a / r_{0}\right)$ is accomplished for the plaquette action by the Necco-Sommer relation [15].

In Table 1 we give numbers for the quantity $\left\langle\left(\alpha_{s} / \pi\right) G G\right\rangle$ for finite $L$ and boosted perturbation theory at $L=\infty$. For the perturbative values at finite $L$ we use the hypergeometric model as given 
in (3.3). The table shows that the boosted result for extrapolation $L=\infty$ agrees within errors with the model value at finite lattice size $L=12$. The given errors have been estimated by varying the fit window $\beta_{\min } \leq \beta \leq \beta_{\max }$. Our reference value for the gluon condensate is that for $L=\infty$ and

\begin{tabular}{|c|c|c|c|}
\hline$L$ & $r_{0}^{4}\left\langle\frac{\alpha_{s}}{\pi} G G\right\rangle$ & $\left\langle\frac{\alpha_{s}}{\pi} G G\right\rangle\left[\mathrm{GeV}^{4}\right]$ & fit range \\
\hline 6 & $1.08(10)$ & $0.034(3)$ & $5.73 \leq \beta \leq 6.00$ \\
8 & $1.22(11)$ & $0.039(4)$ & $5.78 \leq \beta \leq 6.10$ \\
12 & $1.29(17)$ & $0.041(6)$ & $5.78 \leq \beta \leq 6.27$ \\
\hline$\infty$, boosted & $1.33(7)$ & $0.042(2)$ & $5.78 \leq \beta \leq 6.17$ \\
\hline
\end{tabular}

Table 1: Gluon condensate at finite $L$ for Wilson gauge action $\left(r_{0}=0.467 \mathrm{fm}\right)$. The last column shows the used $\beta$-fit range.

boosted perturbation theory given in Table 1 . With the chosen $r_{0}$ it is larger than the value obtained by sum rule calculations [1].

\section{Acknowledgements}

This investigation has been supported partly by DFG under contract SCHI 422/8-1. We thank the RCNP at Osaka university and the HLRN Berlin/Hannover for providing computer resources.

\section{References}

[1] M. A. Shifman, A. I. Vainshtein and V. I. Zakharov, Nucl. Phys. B 147 (1979) 385.

[2] T. Banks, R. Horsley, H. R. Rubinstein and U. Wolff, Nucl. Phys. B 190 (1981) 692.

[3] A. Di Giacomo and G. C. Rossi, Phys. Lett B 100 (1981) 481.

[4] J. Kripfganz, Phys. Lett. B 101 (1981) 169; R. Kirschner, J. Kripfganz, J. Ranft and A. Schiller, Nucl. Phys. B 210 (1982) 567.

[5] E.-M. Ilgenfritz and M. Müller-Preussker, Phys. Lett. B 119 (1982) 395.

[6] R. Alfieri, F. Di Renzo, E. Onofri and L. Scorzato, Nucl. Phys. B 578 (2000) 383 [arXiv:hep-lat/0002018].

[7] F. Di Renzo and L. Scorzato, JHEP 0110 (2001) 038 [arXiv: hep-lat/ 0011067 ].

[8] P. E. L. Rakow, PoS LAT2005 (2006) 284 [arXiv: hep-lat/ 0510046 ].

[9] Y. Meurice, Phys. Rev. D 74 (2006) 096005 [arXiv: hep-lat / 0609005 ].

[10] S. Narison and V. I. Zakharov, Phys. Lett. B 679 (2009) 355 [arXiv: 0906.4312 [hep-ph] ].

[11] P. Weisz, Nucl. Phys. B 212 (1983) 1.

[12] F. Di Renzo and L. Scorzato, JHEP 0410, 073 (2004) [arXiv: hep-lat/ 0410010 ].

[13] R. Horsley et al., in preparation.

[14] R. Horsley, P. E. L. Rakow and G. Schierholz, Nucl. Phys. Proc. Suppl. 106 (2002) 870 [arXiv:hep-lat/0110210].

[15] S. Necco and R. Sommer, Nucl. Phys. B 622 (2002) 328 [arXiv: hep-lat/0108008]. 